www.mdpi.com/journal/sensors

\title{
Performance assessment of a fast temperature sensing system based on bare FBGs and fast spectrum
}

\section{analyzer}

\author{
Wei Chen, Guido Perrone and Alberto Vallan \\ Politecnico Di Torino, Corso Duca degli Abruzzi, 24 - 10129 Torino, ITALY; \\ e-mails: name.surname@polito.it.
}

Published: 1 June 2014

\begin{abstract}
The paper presents a fast all-optical temperature sensing system that can be used as a fiber optic replacement of thermocouples when localized measurements with small time constants are required, such as in the thermal ablation of some tumor cells. Fast response and point measurement capability have been obtained thanks to the combination of a fast spectrum analyzer and reduced footprint sensors. The measurement architecture is quite conventional, being built around the broadband source interrogation approach, but uses a specific spectral fitting method with Gaussian functions to improve the measurement resolution of the spectrometer from about $10{ }^{\circ} \mathrm{C}$ to about $0.1{ }^{\circ} \mathrm{C}$, without adding too much of computation complexity and therefore spoiling the spectrometer speed performance. Then, aiming at applications in the medical field where a non-metallic temperature probe with small dimensions and fast response is required, transducers based on bare fiber Bragg gratings have been built and characterized to critically assess their performance.
\end{abstract}

Key words: Fast Temperature Measurement; Fiber Optical Sensors; Fiber Bragg Gratings.

\section{Introduction}

Fiber Bragg Grating (FBG) sensors are the most widely used fiber optical sensors for temperature, strain and displacement measurements in civil, chemical and 
biochemical engineering [1-3] because of their well mastered and repeatable fabrication technology, combined with high sensitivity, light weight and electromagnetic immunity. Moreover, FBG can be easily multiplexed along a single fiber span to realize a quasi-distributed multipoint sensing system. Another advantage of FBG is that the measurement signal is encoded into the wavelength; so it is very robust to intensity noise, a property highly appreciable in conditions that require long fiber spans to remote the measurement system or when sharp bends in the fiber are necessary to reach the measurement point [4]. Measurement signal wavelength encoding, however, represents also a drawback because it makes the sensor interrogation system more complex: in a standard FBG the peak wavelength shifts by about $10 \mathrm{pm}$ for a $1{ }^{\circ} \mathrm{C}$ temperature variation; so, a wavelength resolution in the order of the picometer is required to obtain a temperature resolution comparable to that of other commonly used sensors, such as thermocouples and electronic devices. Such a spectral resolution, however, is well below the capabilities of typical reasonably low-cost and portable spectral measurement systems (here bench-top lab equipment is not considered), which have a resolution limited to hundreds of picometers.

Indeed, most recent commercial FBG interrogators for in-field sensing applications mainly adopt Charge-Coupled Device (CCD) spectrometers [5], which provide quite a fast response (up to few kilohertz), but present a CCD resolution limited to about 100 pm, a value 50 times larger than what would be desirable.

Then, some temperature measurement applications are even more critical since they not only require high resolution, but also a miniaturized transducer and with a very short time constant. An example of such critical operating environment is radiofrequency (RFA) or microwave ablation (MWA) of tumors in the liver [6]; in this case a localized heat is generated through a needle to kill the tumor cells, and a precise control of the instantaneous temperature in reference points is necessary to properly calibrate the treatment. Thermocouples, which are known for their small size and fast response, cannot be used due to the presence of radiofrequency, as for all other metallic devices, but fiber Bragg gratings could provide a valid all-dielectric alternative. However, typical commercial FBG temperature sensors are designed for civil or industrial applications and are mounted inside large packages, moreover specifically designed to increase the sensitivity, but at the expenses of the response time constant.

In this paper we propose a temperature sensing system able to overcome these limitations by using a bare FBG as the sensing component, and a spectral fitting procedure to improve the resolution of standard spectrometers without increasing the scanning time.

\section{Experimental Section}

\subsection{Working principle}

Fiber Bragg gratings are obtained by inscribing a periodic refractive index modulation into the core of single-mode optical fibers. They present a notch filter response, with the peak reflected wavelength - the so-called Bragg wavelength, $\lambda_{\mathrm{B}}-$ that depends on the modal effective index and on the period of index perturbation. When used as 
sensors, FBGs transduce the variation of the physical quantity under measure into a change of the Bragg wavelength, either directly (as in the case of temperature or strain, which directly act on the refractive index and on the perturbation period) or indirectly, typically through the application of a stress to the fiber.

The variation of the Bragg wavelength with the temperature and strain variation can be expressed as:

$$
\frac{\Delta \lambda_{\mathrm{B}}}{\lambda_{\mathrm{B}}}=\mathrm{K}_{\mathrm{T}} \cdot \Delta \mathrm{T}+\mathrm{K}_{\varepsilon} \cdot \Delta \varepsilon
$$

where, $\Delta \lambda_{\mathrm{B}}$ is the variation of Bragg wavelength, $\mathrm{K}_{\mathrm{T}}$ is the temperature sensitivity, $\mathrm{K}_{\varepsilon}$ is the strain sensitivity, $\Delta \mathrm{T}$ is the temperature variation, $\Delta \varepsilon$ is variation of the strain.

Since in the experiment reported in the following, the FBG sensor does not undergo changes in the applied strain, the only relevant effect is the variation induced by the change of temperature. For variations of the Bragg wavelength $\Delta \lambda_{\mathrm{B}}$ less than $1 \mathrm{~nm}$, negligible with respect to the Bragg wavelength, which is $1530 \mathrm{~nm} \sim 1550 \mathrm{~nm}$, it is reasonable to assume that $\mathrm{K}_{\mathrm{T}} \lambda_{\mathrm{B}}=\mathrm{C}_{\mathrm{T}}$, called temperature coefficient, is constant during the whole experiment. Then the formula to describe the relation between the Bragg wavelength and the temperature $\mathrm{T}$ becomes:

$$
\lambda_{\mathrm{B}}=\mathrm{C}_{\mathrm{T}} \cdot \mathrm{T}+\lambda_{\mathrm{o}}
$$

where $\lambda_{o}$ is the Bragg wavelength at $0 \mathbb{C}$.

\subsection{Sensor Characterization}

The developed sensing system uses bare fiber gratings in order to reduce the sensor time constant; however, since bare gratings are not typically used for temperature sensing, their temperature coefficient is not reported in data sheets with the required accuracy, so a proper preliminary characterization is necessary before their practical application. This has been carried out using a climatic chamber programmed to change the temperature in the range $20{ }^{\circ} \mathrm{C}$ to $55^{\circ} \mathrm{C}$. During the test, the $\mathrm{FBG}$ response has been recorded and the actual temperature has been measured using a reference temperature sensor (LM35) having a linearity of about $0.25{ }^{\circ} \mathrm{C}$ [7]. The reference sensor acts here as a standard and, therefore, its accuracy has not been taken into account.

The FBG has been interrogated using an SLED and a fast spectrometer. Then, in order to control the spectrometer, a data acquisition and processing system has been implemented in Labview ${ }^{\mathrm{TM}}$. The architecture of the experimental set-up is reported in Fig. 1. 

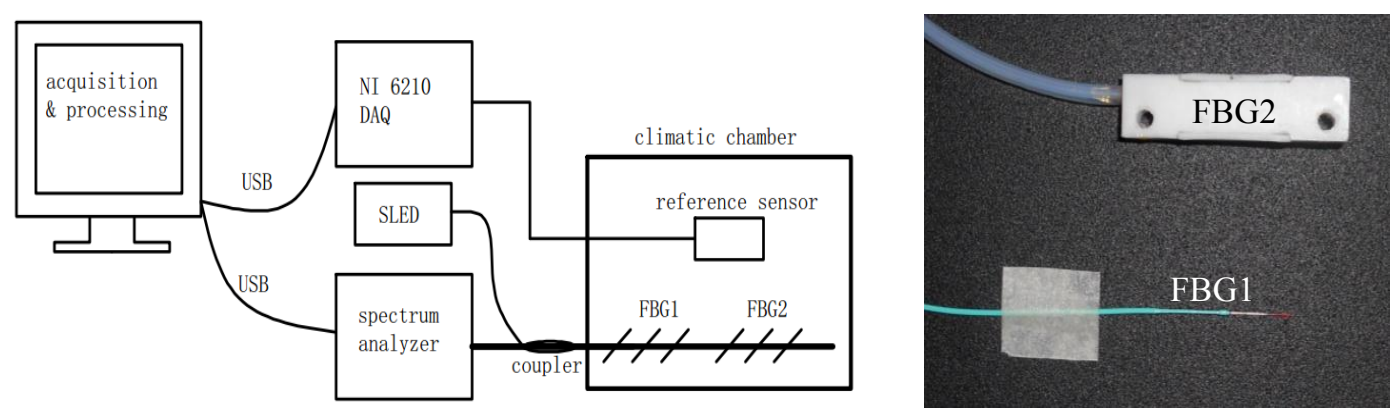

Figure 1. The set-up for the characterization and a detail of the bare FBG (FBG1) and of the commercial FBG temperature sensor (FBG2)

For comparison purposes, a second FBG sensor specifically designed for temperature measurements (FBG2 in Fig.1) has been interrogated as well during the characterization tests. This sensor is packaged in a plastic container.

The spectrometer resolution is due to the CCD sensor resolution and it is limited to $0.1 \mathrm{~nm}$, which corresponds to a temperature resolution of about $10^{\circ} \mathrm{C}$, a value that is larger than what is expected for the proposed application. Therefore, the resolution has been then increased using a spectral fitting algorithm based on Gaussian function. The fitting procedure has been written in Matlab ${ }^{\mathrm{TM}}$, and then embedded in the Labview $^{\mathrm{TM}}$ program. Thanks to the data fitting procedure a resolution of about $1 \mathrm{pm}$, corresponding to about an error of about $0.1 \mathrm{C}$, has been obtained.
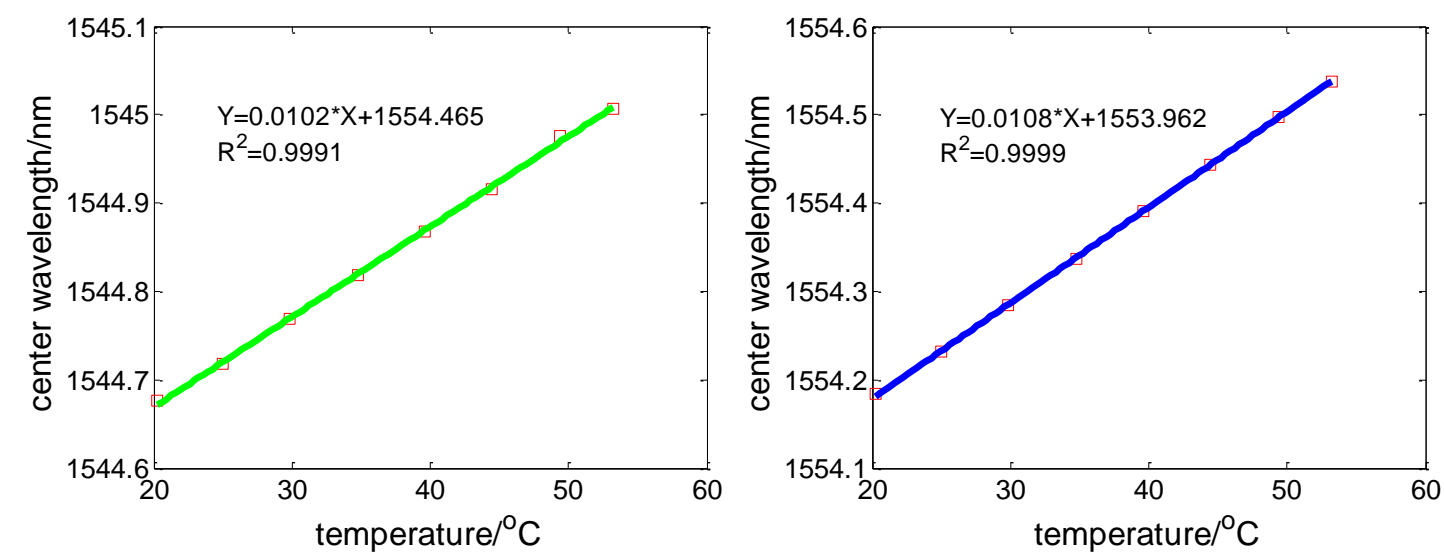

Figure 2. Characterization results for bare FBG (FBG1, left) and commercial FBG (FBG2, right).

The FBG temperature coefficient has been obtained by fitting the variation of the Bragg wavelengths with a linear model. The characterization results are shown in Fig.2, where is possible to see that both the FBG sensors present good linearity and a temperature coefficient close to about $0.01 \mathrm{~nm} /{ }^{\circ} \mathrm{C}$. The temperature error computed, after the characterization, as the difference between the linear model and the reference thermometer is of about $0.1{ }^{\circ} \mathrm{C}$ for FBG2 and about $0.3{ }^{\circ} \mathrm{C}$ for the bare FBG. The reason for the larger error of the bare grating is mainly due uncontrollable strains and 
bending, whereas the package of the commercial one isolates the sensing part from external stress.

\subsection{Preliminary results}

After the FBGs have been characterized, they have been removed from the climatic chamber and employed to measure the ambient temperature for about 4 h. Fig. 3 reports the temperature measured using FBG1 and FBG2. A comparison with the reference thermometer (not shown in the Figure) has shown that the bare FBG presents a temperature offset of about $0.2{ }^{\circ} \mathrm{C}$. This offset is due to the sensor strain and it is almost constant during the test, and it acts as change in the initial Bragg wavelength $\lambda_{o}$ of Eqn. 2. The offset has been then compensated, obtaining the green curve in Fig. 3, which is now in good agreement with the commercial FBG and proving that the sensor sensitivity - the temperature coefficient in Eqn. 2 - does not change significantly.

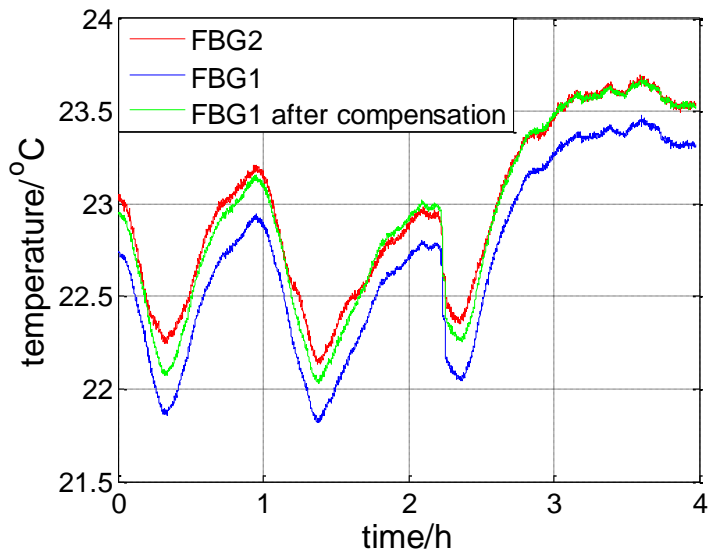

Figure 3. Comparison test between the bare FBG (FBG1) and the commercial FBG (FBG2).

A further test has been carried out in order to assess the thermal time constant of the bare FBG. To this aim, the sensor has been immersed in a container filled with a mix of ice and water and the temperature evolution has been recorder. A different reference thermometer based on a miniature T-type thermocouple has been here employed in this test since the LM35 has a time constant larger than $1 \mathrm{~s}$.
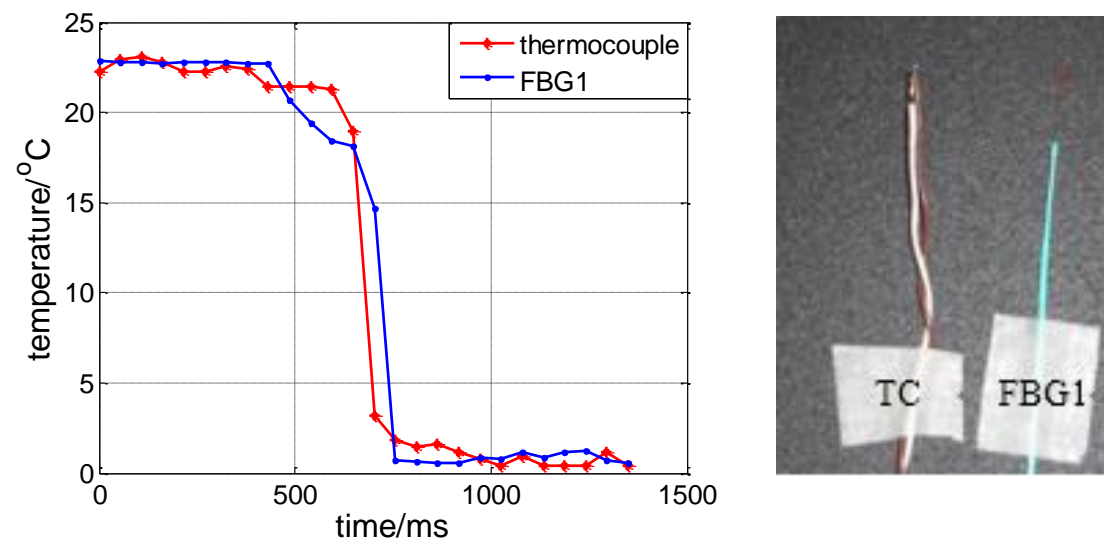

Figure 4. Time constant comparison between the thermocouple and bare FBG (FBG1) 
Fig. 4 shows the sensor responses in the presence of a sharp temperature change from ambient temperature to about $0{ }^{\circ} \mathrm{C}$. In this preliminary test the bare $\mathrm{FBG}$ has shown a time constant of about $400 \mathrm{~ms}$, shorter that the thermocouple response.

\section{Conclusions}

In this paper, we have proposed and preliminary characterized a temperature sensing system based a bare FBG and a fast spectrum analyzer. The sensor is able to measure fast temperature changes with a good resolution even though, being unprotected, it is sensitive to external strain and thus requires compensation when an absolute temperature measurement has to be performed. The system presents a high acquisition rate, a small sensor footprint and electromagnetic immunity, characteristics promising which for potential applications in the medical field where a non-metallic temperature probe with small dimensions and fast response is required, such in temperature measuring during radiofrequency (RFA) or microwave ablation (MWA) of tumors.

\section{Conflicts of Interest}

The authors declare no conflict of interest.

\section{References}

[1] H.C Fan; J.W, Qian; Y.N Zhang etc. “A Novel FBG Sensors Network for Smart Structure Vibration Test". Proceedings of the 2007 IEEE, International Conference on Robotics and Biomimetics, December 15 -18, 2007, Sanya, China.

[2] P.Boland; G. Sethuraman; A.Mendezb; T.Graverc etc. "Fiber Bragg grating multi-chemical sensor". Proc. of SPIE, 2005, Vol. 5994 599407, 1-7.

[3] S. Keren; M. Horowitz. "Distributed three-dimensional fiber Bragg grating refractometer for biochemical sensing". OPTICS LETTERS, America, 2003; Vol. 28, No. 21, 2037-2039.

[4] Montserrat, F.V.; Sergio, R.R.; Manuel, L.A.; "Remote $(250 \mathrm{~km})$ Fiber Bragg Grating Multiplexing System”. Sensors 2011, 11, 8711-8720.

[5] J.Alves; J.L. Santos; A.Carvalho; A.Lage; "Fiber Bragg sensor interrogation system based on a CCD spectrometer". Proceedings of IEEE, 2003, Vol.2, 909-913

[6] American Cancer Society, http:// www.cancer.org/cancer/ livercancer/ detailedguide/liver-cancer-treating-tumor- ablation.

[7] Texas Instruments, http://www.ti.com/lit/ds/symlink/lm35.pdf.

(C) 2014 by the authors; licensee MDPI, Basel, Switzerland. This article is an open access article distributed under the terms and conditions of the Creative Commons Attribution license (http://creativecommons.org/licenses/by/3.0/). 